\title{
1 Using historical museum samples to examine divergent and parallel evolution in the invasive
}

2 starling

3 Katarina C. Stuart ${ }^{1}$, William B. Sherwin ${ }^{1}$, Jeremy J. Austin ${ }^{2}$, Melissa Bateson ${ }^{3}$, Marcel Eens ${ }^{4}$, Matthew

C. Brandley ${ }^{5,6}$, Lee A. Rollins ${ }^{1}$

5

${ }^{1}$ Evolution \& Ecology Research Centre, School of Biological, Earth and Environmental Sciences, UNSW

Sydney, Sydney, New South Wales, Australia

7

${ }^{2}$ Australian Centre for Ancient DNA (ACAD), School of Biological Sciences, University of Adelaide,

8

Adelaide, SA, Australia

9

${ }^{3}$ Institute of Neuroscience, Newcastle University, Newcastle upon Tyne, UK

Wilrijk, Belgium 


\section{Abstract:}

During the Anthropocene, Earth has experienced unprecedented habitat loss, native species decline,

and global climate change. Concurrently, greater globalisation is facilitating species movement, increasing the likelihood of alien species establishment and propagation. There is a great need to understand what influences a species' ability to persist or perish within a new or changing environment. Examining genes that may be associated with a species' invasion success or persistence informs invasive species management, assists with native species preservation, and sheds light on important evolutionary mechanisms that occur in novel environments. This approach can be aided by coupling spatial and temporal investigations of evolutionary processes. Here we use the common starling, Sturnus vulgaris, to identify parallel and divergent evolutionary change between contemporary native and invasive range samples and their common ancestral population. To do this, we use reduced-representation sequencing of native samples collected recently in north-western Europe and invasive samples from Australia, together with museum specimens sampled in the UK during the mid- $19^{\text {th }}$ Century. We found evidence of parallel selection on both continents, possibly resulting from common global selective forces such as exposure to pollutants (e.g. TCDD) and food carbohydrate content. We also identified divergent selection in these populations, which might be related to adaptive changes in response to the novel environment encountered in the introduced Australian range. Interestingly, signatures of selection are equally as common within both invasive and native range contemporary samples. Our results demonstrate the value of including historical samples in genetic studies of invasion and highlight the ongoing and occasionally parallel role of adaptation in both native and invasive ranges. 


\section{Introduction:}

47
The ecological and economic impacts of invasive species are a growing concern in our globalised world. Increased intercontinental travel and trade is giving rise to new or reinforced invasion pathways (Turbelin et al. 2017), resulting in a great number of alien species becoming established and spreading within novel ranges (Hulme 2009). The financial cost of invasive species within Australia is estimated in excess of 13 billion dollars annually (Hoffmann \& Broadhurst 2016). With habitat clearing and climate change expected to favour invasive species over native ones, the environmental and financial cost of invasive species is only expected to rise in the future (Dukes \& Mooney 1999). Many studies on invasive species' success involve examining evolutionary changes following introduction and focus on rapid adaptation to novel environments (Prentis et al. 2008). This information is vital for long term management of invasive populations.

Understanding evolutionary trends across a species' native and invasive ranges will help determine important adaptive elements that aid species' persistence in a changing world. Species that are invasive present a contrariety when they face population decline within their native range (Rogers et al. 2006; Delibes-Mateos et al. 2009; Erfmeier \& Bruelheide 2010; Bishop 2011). Research efforts should tackle ecological questions of conservation and invasion management concurrently, enabling us to understand how and why patterns of adaptation in a species' native and invasive populations may differ. It is possible that the translocation and establishment process itself may select for traits that enable an individual to overcome otherwise detrimental environmental instability or other novel stressors, increasing general fitness (Callaway \& Ridenour 2004; Liu \& Trumble 2007). Understanding how the invasion process may induce differences in population persistence is made even more pressing by the increasing anthropogenic impact on the natural world, including ongoing land alteration, environmental contamination, and human induced climate change (Hellmann et al. 2008). 

invasive populations (Hofmeister et al. 2021a). However, such approaches exclude the temporal element of species' change, so that such studies assume native populations have not changed since the founders of the invasive population were collected. This would then lead to the conclusion that all similarities between native and invasive populations result from a common ancestral population and are not due to parallel change since separation. However, with global anthropogenic change impacting the natural world, it is reasonable to assume that altered or increased selective pressures have arisen during the post-industrialised world, shaping species world-wide (Sokolova \& Lannig 2008; Siepielski et al. 2017). Historical specimens therefore provide an unparalleled tool to better contextualise divergent versus parallel evolution, providing phenotypic and, more recently, genotypic information that can be used to identify temporal changes in species ranges and traits (Ewart et al. 2019; Lopez et al. 2020). Studies focusing on rapid local adaptation in invasive species may now make use of historic DNA alongside contemporary samples to understand the selective forces shaping both invasive and native ranges concurrently. samples to investigate both divergent and parallel genetic change within an invasive species. The establishing on every other continent barring Antarctica (Higgins et al. 2006). Despite this, the native range starlings are themselves a conservation focus, with declines of more than $50 \%$ in some countries (Versluijs et al. 2016) putatively associated with shifts in farming practice that are common in their native range (Freeman et al. 2007; Heldbjerg et al. 2016). Fortunately, due to the historic popularity of collecting bird skins, historical starling samples may be found scattered across many museums and institutions in both their native range and within invaded countries. These skins serve as untapped reserves of genetic information, which may be used to track temporal genetic changes 

in selective forces.

across the native range, reveal information regarding historical population structure, and provide context that enables us to better understand current patterns of native range starling decline.

Starlings present a prime example of how the combination of data from invasive, native, and historical populations can clarify our understanding of evolution in both native and invasive contexts. Introduced into Australia in the 1860 's, the starlings' range now stretches across the continent's eastern and southern coasts (Long 1981). Genetic analysis supports strong population substructuring across the invasive Australian range (Rollins et al. 2009, 2011), with reducedrepresentation sequencing data indicating the two main subpopulations likely resulted from allelic differences in founding populations at different introduction sites (Stuart \& Cardilini et al. 2021a). The historical specimens available for this species were collected within 15 years of the earliest documented introductions to Australia in 1856 (Long 1981), providing a snapshot of native starling populations at the time when founders were transported to Australia.

To better understand patterns of population structure and signatures of selection present in the invasive Australian range, we used a reduced-representation sequencing approach to compare contemporary Australian (AU) and native range (United Kingdom, UK; Belgium, BE) starlings to historical UK samples collected during the period when the Australian founders were collected. Moreover, this project explores proximate drivers of invasive species' evolution in the face of novel selection provided by new environments. Specifically, we compare population structure of native and invasive contemporary S. vulgaris samples, and we explore genomic divergence between contemporary and historical S. vulgaris and assess the putatively adaptive capacity of these genomic changes. Finally, we use historic samples as a basis of comparison to determine regions of parallel change in both the contemporary native and invasive populations to better understand global shifts 
2. Methods:

\subsection{Sample collection and extraction (historical starlings):}

121 We sourced historic starling specimens (HS) from the Natural History Museum (NHM) in Tring, UK

$122(\mathrm{~N}=15)$. Historical samples were selected on the basis of sample quality, completeness of collection record, and sample collection date (samples collected from 1857-1871, during the period when the Australian introductions took place; Higgins et al. 2006; Supplementary Materials, Table S1). buffer (+0.05\% Tween-20) for a final elution volume of $80 \mathrm{uL}$.

\subsection{Sample collection and extraction (contemporary starlings):}

131 We sourced contemporary native range starling samples from two UK locations: Monks Wood (MW: 

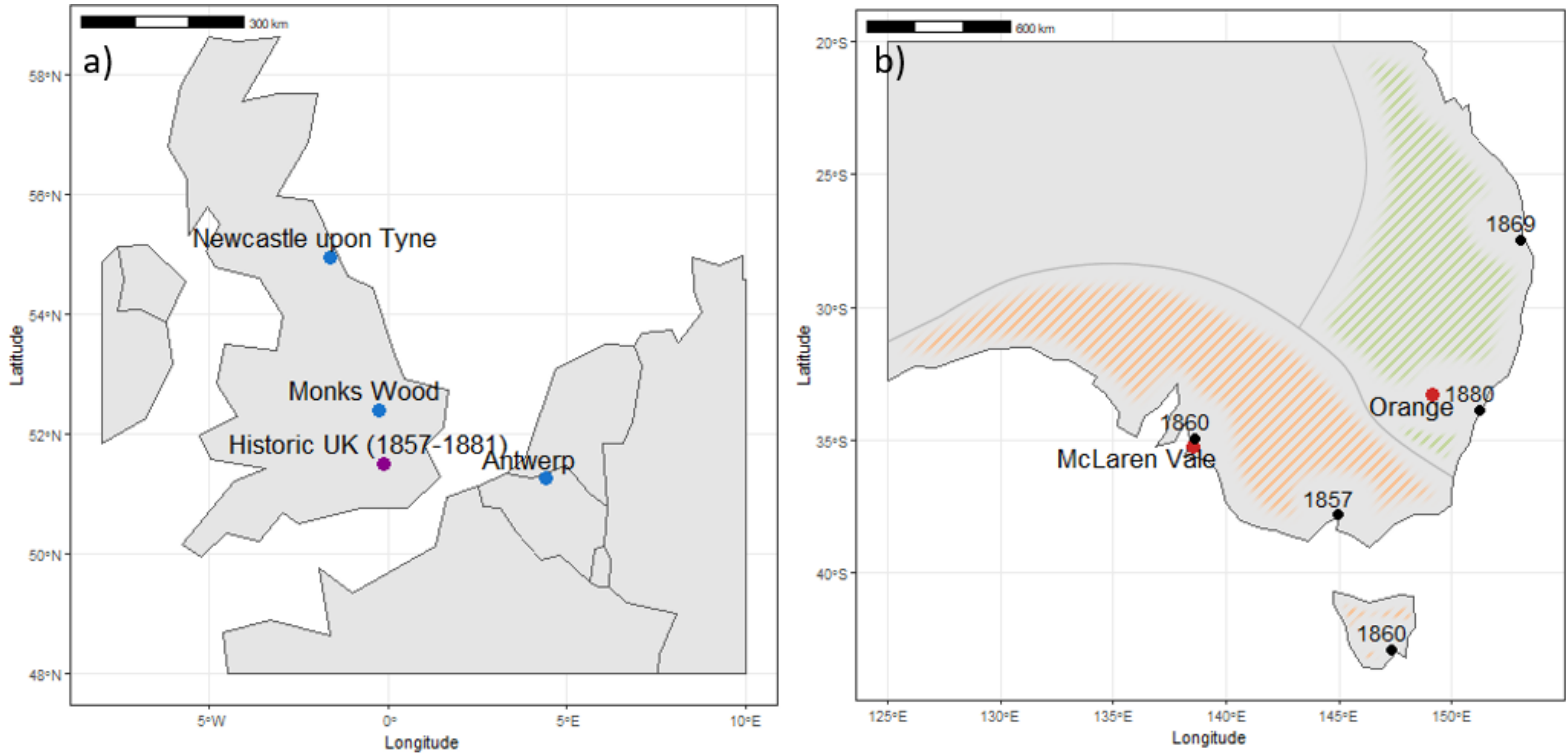

Figure 1: Geographical distribution of the Sturnus vulgaris collection sites in the United Kingdom and Belgium (native range, blue and purple), Australia (invasive range, red), and historical samples. The coloured shading on the Australian map denotes their Australian range, broken up into the two main subpopulations. Introduction sites are marked in black on the Australian map, with first introduction year listed adjacently.

\subsection{Sequencing and genome variant calling:}

We sequenced 75 contemporary and 15 historical samples using the DArTseq protocol (Kilian et al.

2012), using a restriction enzyme double digest of Pstl-Sphl. The sequencing was conducted on a

Hiseq 2500 , producing $312,907,523$ single-end reads of raw data across the 90 samples $(26,408,649$

across the 10 successfully sequenced historical samples; Supplementary Materials, Table S1).

We used the STACKS v2.2 (Rochette et al. 2019) pipeline (Rochette \& Catchen 2017) to process the DArTseq raw data into variant calling format (vcf). We used the process_radtags function to clean the tags; discarding reads of low quality (-q), removing reads with uncalled bases (-c), and rescuing barcodes and radtags (-r). We used the Burrows-Wheeler aligner (BWA) v0.7.15 (Li \& Durbin 2009) aln function to align the read data to the reference genome S. vulgaris vAU1.0 (Stuart et al. 2021b). Using FastQC, we identified base sequence bias in the adapter region, and so the first five base sequences were trimmed (-B 5) during alignment. We used the BWA samse function to produce 
We ran the cleaned data through STACKS gstacks (default parameters) and then populations to call

158

159

160

161

162

163

164

165

166

167

168

169

170

171 the SNPs.

We produced an unfiltered SNP data set by running STACKS populations with no parameter

thresholds specified. We used this data set to produce the unfiltered loci and site counts

(Supplementary Materials, Table S2), split the data into three separate files for further assessment of historic sequencing data (contemporary native range samples: MW, NC, and AW; Australian samples: OR and MV; and historic samples; HS). We calculated variant base substitutions in VCFSTATS v0.0.5 (Lindenbaum 2015) and variant density mapped along the reference genome scaffolds using SAMTOOLs bedtools function (window size 1,000,000bps). We used the DARTR V1.1.11 (Gruber et al. 2018) function gIPlot to create a smear plot of the mapped variant data across individuals and genomics sites. This resulted in a population genetic file of 239,538 SNPs. We also passed the raw single-end read data through the Stacks BWA-mem, and the bowtie-GATK, variant calling pipeline (Supplementary Material: Appendix 1), to compare the quantity of site variant data that was successfully mapped and assess how these alternate variant calling software performed for reduced representation sequencing data sets that contained degraded historical DNA.

We generated a 'population genetics' variant file by running STACKS populations, filtering for a minimum per-population site call rate of $50 \%(-r 0.5)$, a minimum populations per-site of $2(-p 2)$, a minimum loci log likelihood value of -15 (--Inl_lim -15), with one random SNP per locus retained (-write_random_snp). We used VCFTooLs v0.1.16 (Danecek et al. 2011) to filter the following parameters: maximum missingness per site of $50 \%$ (-max-missing 0.5$)$, minor allele frequency of $2.5 \%$ (MAF; --maf 0.025), minimum loci depth of 5 (--minDP 5), minimum genotype quality score of 15 (-minGQ 15) and site Hardy-Weinberg Equilibrium exact test minimum $p$ value 0.001 (--hwe 0.001). We chose a moderate high threshold for missingness as over-filtering missingness has been found to influence population structure in reduced representation data sets (Wright et al. 2019; Selechnik et 

of which are important for capturing neutral population substructure (HWE SNPs were however left in for ADMIXTURE analysis (Deng et al. 2001)). After filtering, we checked individual relatedness, and closely related individuals were removed so that there was only one representative from each familiar cluster in the final data (Supplementary Materials: Fig. S1). This resulted in a population genetic variant file of 23,683 SNPs using in the below section 'Population structure analysis' $(25,572$ variant sites if HWE were included).

We generated a 'selection' variant file by using STACKS populations to align the raw reads (with --Inl_lim -15 flag), and then used VCFTOOLs to filter out only reads present in at least $50 \%$ of the historic individuals (i.e. in at least 5 historic individuals), with additional quality filtering (--minGQ 15 -minDP 2), resulting in 12,219 SNP sites. Only these sites were then retained to filter the original populations variant file, along with a MAF minimum of $2.5 \%$ to remove possible sequencing errors. This produced a data set with fewer SNPs than the population genetics variant file but retained only SNPs sequenced in a moderate number of historic individuals, which would be necessary for selection analysis. We filtered the selection variant file to form three pairwise population SNP data comparisons; UK-HS (UK populations MW, NC, BE and 10 historic individuals), AU-HS (AU populations populations $\mathrm{MV}$ and $\mathrm{OR}$ ). While the native range population may contain a mix of resident and migratory individuals, because we see minimal population structure in the native range and very small $F_{s r}$ values $(0.003-0.008)$ we decided to include all contemporary native range samples in this analysis. Within each of these three variant file subsets, we retained SNP sites present in at least 5 contemporary individuals, as the file was already filtered for loci present in at least $50 \%(5 / 10)$ of historic individuals. This relatively lenient filtering was necessitated by the smaller number genomic 
that was present. This resulted in three pairwise population files used in the below section 'Selection analysis': UK-HS (4997 SNPs), AU-HS (5006 SNPs), and UK-AU (4957 SNPs).

\subsection{Population structure analysis:}

We analysed the population genetics variant file several ways to examine the population structure and differentiation between and within the historical and contemporary sample regions. We used $R v$ 3.5.3 (R Core Team 2017) to run the SNPRELATE snpgdsPCA function to create a principal components analysis (PCA) of the loci. We used ADMIXTURE v1.3.0 to determine individual ancestry proportions for each of the following three sample subsets: all samples, contemporary native range and historic, and contemporary Australian. We calculated marginal likelihood for model complexity (K) 1-8 by averaging over 50 runs, and admixture proportion (Q) profile were generated by CLUMPAK (Kopelman et al. 2015) (run on default settings) to obtain an average $Q$ profile from 25 runs. We used the DARTR function gl.dist.pop to calculate the Euclidean distance between sampling locations, and the STAMPP function stamppFst to calculate pairwise $F_{S T}$ between sampling locations (nboots=1, percent=95, nclusters=1). Finally, we used the DARTR function gl.tree. $n j$ to visualise phylogeny of the 6 sampling groups.

\subsection{Selection analysis:}

When looking for diversifying selection, allele frequency based approaches are often used, such as BAYESCAN v2.1 (Foll \& Gaggiotti 2008). BAYESCAN aims to identify SNPs subject to natural selection by assigning a per-site posterior probability estimated by comparison of explanatory models with and without selection. We conducted BAYESCAN SNP outlier analysis, with prior odds for the neutral model set to 10 (-pr_odds 10), and a false discovery rate (FDR) of 0.05 .

Because the smaller sample sizes in this selection analysis lack statistical power, and BAYESCAN uses stringent criteria to flag selection, the alpha values produced by BAYESCAN were used to identify additional putative sites under selection. The alpha value is the locus-specific component of 
selection identified in the BAYESCAN selection model, where a large positive alpha value indicates diversifying selection, and a large negative value indicates balancing or purifying selection. We retained sites that reported an alpha value of $>0.1$, and then filtered these for a minimum $r 2$ linkage value of 0.05 (window size $1000 \mathrm{bp}$ ) using SAMTOOLs bcftools, similar to the approach used in GloriaSoria et al. (Gloria-Soria et al. 2016). We plotted Alpha values in rank order and chose an alpha SNP as under putative selection will yield more false positives, hence we chose to filter for high linkage. Filtering out sites with low linkage will help select against false positive sites whose allele frequencies (and hence alpha value) appear to indicate selection, because linkage often occurs around a gene under selection (Slatkin 2008). diversity SNPs (Ewart et al. 2019), which may impact single site approaches to examining divergent selection. Therefore, we used a $\mathrm{F}_{\mathrm{ST}}$ sliding window approach to identify genomic regions of putative diversifying selection. VCFTOOLs weir-fst-pop function was used to analyse weighted $\mathrm{F}_{\mathrm{ST}}$ in $900000 \mathrm{bp}$ windows (10000 window step). We chose this window size primarily based on the ratio of variants to genome size, with the chosen windows putatively spanning 3-4 variants, with small step sizes then allowing shifts in $\mathrm{F}_{\text {ST }}$ patterns to be pinpointed more exactly. We selected site windows that reported a weight $F_{S T}$ value in the top 99th percentile for each pairwise population selection analysis and analysed the Fst of SNPs within these windows in rank order. We retained any SNP within these outlier windows that lay above an Fst threshold relevant for each pairwise data set (this was putative outlier SNPs. 
within each pairwise population comparison, to be used for further variant analysis.

\subsection{Variant analysis and annotation}

SNPs that were reported as outliers across either the UK-HS or AU-HS data set, as well as the UK-AU data set, we designated as sites under divergent selection. SNPs that were reported as outlier across both UK-HS and AU-HS data sets we designated as sites under parallel selection. The remaining outlier SNPs we identified only in one dataset: UK-HS (putative UK selection); AU-HS (putative AU selection); or UK-AU (putative UK-AU divergence). Further details are given below in section 3.4 Putative adaptive selection.

We analysed these five groups of SNPs for their functional roles and the nature of the mutation. We completed SNP analyses primarily using VARIANT EFFECT PREDICTOR (VEP) (McLaren et al. 2016), using the genome annotation version released alongside the $S$. vulgaris vAU1.0 assembly (Stuart et al. 2021b) to examine the functional consequences of the SNPs (processed to exclude multiple isoforms using AGAT agat_sp_keep_longest_isoform.pl (Dainat 2020)). We used BEDTOOLS and the AGAT function agat_sp_functional_statistics to extract genes and transcripts that overlapped the putative loci under selection, and extract gene ontology (GO) terms. We used REVIGO (Supek et al. 2011) to visually summarise GO terms, and we calculated allele frequencies at SNP sites using BEDTOOLS.

Lastly, to test if there was an overrepresentation of SNPs located on the macrochromosomes (>20 Mb, as described in Backström et al. 2010) , microchromosomes, or the sex chromosome, we used a Chi-square test to examine overrepresentation of these SNP types across four different SNP groupings: the divergent SNPs (both UK and AU), the parallel SNPs, the SNPs under selection (Putative UK, AU, and UK and AU SNPs under selection), and the remainder of the SNPs that were not flagged as putatively being under selection in any of the pairwise data sets (no selection). We 
analysed these data using the chisq.test() function in R. To ensure results from this analysis were not artifacts of the data (for example due to diploid variant calling on hemizygous ZW females), we conducted two types of analysis of variance (ANOVA) tests. Firstly, we tested to see whether major allele frequency was significantly associated with an interaction between SNPs type (selection vs non-selection) and SNP location (sex chromosome vs autosome). Secondly, we examined whether between SNPs type (selection vs non-selection) and SNP location (sex chromosome vs autosome). We tested for an interaction between SNPs type (selection vs non-selection) and SNP location (sex chromosome vs autosome on either the major allele frequency or number of alleles sequenced for a SNP site using the $\operatorname{aov}($ ) function in R. We constructed this analysis as both major allele frequency and allele counts are employed to flag outliers to the outlier tests used in this study, and an interaction between SNP location and its categorisation as under selection or not would be cause for concern about bias due to data artifacts. This analysis was run with the major allele frequency and allele counts of all individuals, and then separately again for just historic individuals, so see if any of these effects were only affecting historic individuals (e.g. due to smaller sample size, which may make historic allele frequencies more vulnerable to sex chromosome biases).

\section{Results:}

\subsection{Population structure of contemporary and historical S. vulgaris}

Our genetic data revealed strong differentiation between contemporary native and invasive range samples, as well as replicating the previously established subpopulation structure within Australia. Using PCA, the historical samples were genetically intermediate between the contemporary UK and 
samples (Supplementary Materials: Fig. S2c). genetic differentiation from the native range samples than did those from Orange, evident in pairwise $\mathrm{F}_{\mathrm{ST}}$ and Euclidean genetic distance comparisons (Fig. 2c) and corroborated by McLaren Vale admixture proportions lacking the UK cluster (Fig. 2b). The historical samples were found to be most similar to samples from Antwerp, when considering pairwise $\mathrm{F}_{\mathrm{ST}}$ and Euclidean genetic distance (Fig. 2b) and tree analysis (Fig. 2d), while PCA and admixture plots are indicative of more similarity amongst the native range samples by the neighbourhood-joining tree (Fig. 2d). We found that the genetic differentiation between sampling sites across the native range is less than that across the invasive range.

\subsection{Genomic divergence between contemporary and historical S. vulgaris}

319 Using the default BAYESCAN pipeline, we identified a total of 24 outlier loci across the three pairwise comparisons, fifteen of which were found in the UK-AU comparison, eight in the UK-HS comparison, and only one in the AU-HS comparison (Table 1, Supplementary Materials: Fig. S3a-c). Using the 
(Table 1, Supplementary Materials: Fig. S3d-f). Interestingly, we found that across the three pairwise population analyses, not all of the outlier SNPs reported by the BAYESCAN FDR approach were shared with the BAYESCAN alpha+LD approach (Supplementary Materials, Table S3), presumably because they did not meet the linkage criteria. Using the sliding window approach, the AU-HS comparison reported marginally the highest number of putative outlier loci compared to UK-HS and UK-AU (Table approach we also identified in the $\mathrm{F}_{S T}$ sliding window approach, with generally high overlap with the BAYESCAN alpha+LD approach (Supplementary Materials, Table S3). We visualised the outlier sites across all three methods against the starling genome for each of the pairwise population data sets, and they appear to be fairly uniformly spread throughout the genome (Fig. 4).

We pooled putative outlier SNPs across all the analyses done for each pairwise comparison, yielding a total of 71,85 , and 71 unique SNPs across identification methods for UK-HS, AU-HS, and UK-AU respectively (Table 1 ).

\subsection{Putative adaptive selection}

Of the total of 227 SNPs identified as under selection across all three data sets, we found 166 unique SNPs, of which 9 were identified as resulting from divergent selection, and 52 from parallel selection

(Fig. 4a). The remaining SNPs were only present in one of the pairwise data sets, and were categorised as resulting from putative UK selection (16), putative AU selection (27) and putative UK and AU divergent selection (62) (Table 2, Fig. 4a). 
of intergenic variants (Fig. 4d). We found only a few SNPs with predicted protein coding

consequences, with one missense variant present in the UK putative selection SNP list, and one synonymous mutation each in the putative UK selection and putative AU selection SNPs (Fig. 4c and 4d).

While many of the SNPs mapped to unannotated loci, we mapped five SNPs in the divergent data set to annotated genes, along with 26 SNPs in the parallel data set (Table 2, Supplementary Materials; Table S4, Table S5). Only one gene was found to be divergent within the native range (ANKHD1), while four were found to be diverging with the invasive Australian range (GRIK2, Esrrg, ARHGAP10, and Cacna2d3, Supplementary Materials; Table S5). A diverse range of genes were flagged as being under divergent parallel selection in both the native and invasive range, including two that are known to interact with toxins and pollutants (NID2 and SOS1), and two related to carbohydrate processing (SI and INS, Supplementary Materials; Table S5). There was little gene ontology overlap between these two data sets (Supplementary Materials; Fig. S5). was not a proportional distribution of SNPs across macro, micro, and sex chromosomes, $\left(\chi^{2}(6, N=\right.$ $5068)=21.149, p=0.0017)$. We visualised the residuals and contributions, revealing an overabundance of sex chromosome SNPs for both the parallel SNPs as well as the UK, AU and UK and AU SNPs under putative selection (i.e. those SNPs that were reported as an outlier in only one pairwise population comparison; Fig. 6). We conducted additional analysis on potential biases in major allele frequency or allele counts to help validate this result. Across all samples, we found no significant interaction between SNP location (sex chromosome or autosome) and SNP category (under selection or not) for either major allele frequency $\left(F_{1,5064}=0.188\right.$, $p$-value $=0.664$ ) or allele counts $\left(F_{1,5064}=0.278, p\right.$-value $\left.=0.598\right)$. We repeated these analyses for historic individuals only, 
major allele frequency $\left(F_{1,5064}=1.757, p\right.$-value $\left.=0.185\right)$ or allele counts $\left(F_{1,5064}=0.668, p\right.$-value $=$ $0.4136)$.

\subsection{Sequencing and variant calling with historic samples}

Of the 15 historical samples, 10 were successfully sequenced using DArTseq, a success rate (66.7\%;

Supplementary Materials, Table S1) similar to that previous reported (62\%) in a study using museum toe-pad samples ranging from 5-123 yrs old (Ewart et al. 2019). There did not appear to be any trends related to DNA concentration, sample age, or fragmentation between historical tissue samples that were successfully sequenced and those which were not (Supplementary Materials: Table S1; Fig. S6, Fig. S7).

Of the three variant calling pipelines (Supplementary Materials, Table S2), we found that Bowtie2-GATK reported the highest percentage of successfully mapped reads over both contemporary and historical samples, followed closely by BWA-Mem with BWA-Aln having much lower mapped read percentages. However, Bowtie2-GATK reported the smallest numbers of variant sites in the unfiltered and filtered data set, with BWA-Mem reporting slightly higher values than BWA-Aln. These results are in alignment with previous assessments of these software performances for reads of approximately this length (Li \& Durbin 2009; Li 2013). BWA-aln is generally reported to map more conservatively than BWA-mem (Robinson et al. 2017), leading to the much smaller mapped reads percentage, but this did not have a very large effect on the site counts or missing data per individual. The biggest difference between these two was the difference between the number of filtered variant sites for the historical samples, indicating that the lower quality reads produced by the historical samples were most impacted by the change in aligning algorithm. BWA-Aln was used as the variant calling pipeline in this paper because our read length fell on the border of what was recommended for BWA-Aln and BWA-Mem (70 BP), and a more conservative mapping and variant 
calling approach is suitable for population and selection analysis (when approaches are based on persite allele frequencies).

We assessed the unfiltered data, and the base substitution plots per-population revealed that though the historical samples reported lower SNP counts, base substitution frequencies were similar across the three population groupings (Supplementary Materials: Fig. S8a-c). When we mapped and aligned these reads to the genome assembly alongside Illumina whole genome variant data for the species (Hofmeister et al. 2021a), similar patterns are seen between the two sequencing approaches, and across all three population groupings, though with lower resolution in the historical individuals (Fig. 5). Our sequencing and mapping of the historical samples indicate that, despite the lower quality and fragmented DNA, the overall patterns of base substitutions resembled that of the higher quality fresh tissue used from the contemporary populations, and that the reduced representation approach reflected variant densities seen in whole genome sequencing analyses. A smear plot of data revealed that missing data are relatively evenly spaced along the genome for historical samples (Supplementary Materials: Fig. S9), and not centred on particular genomic regions 
a)

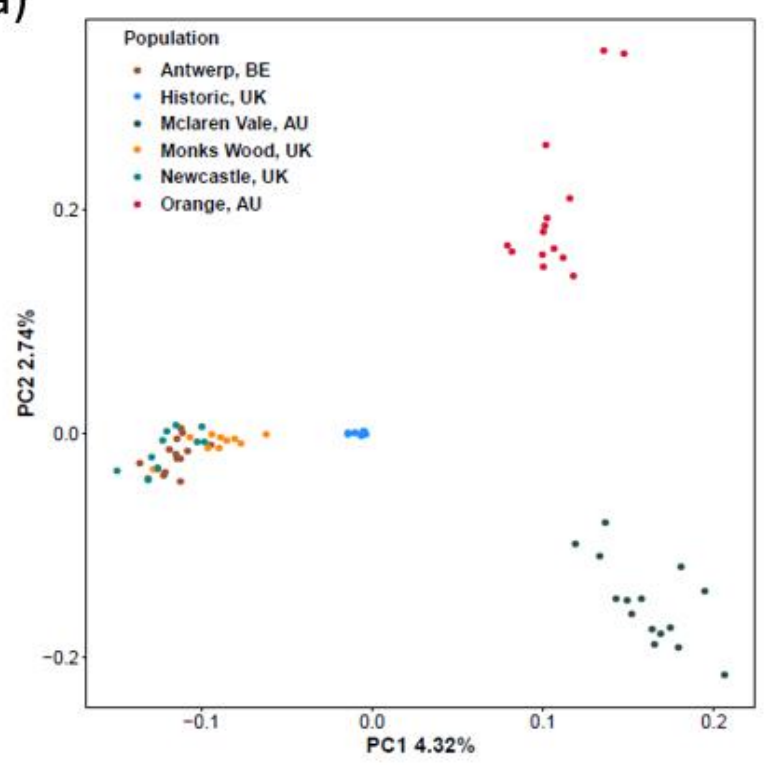

c)

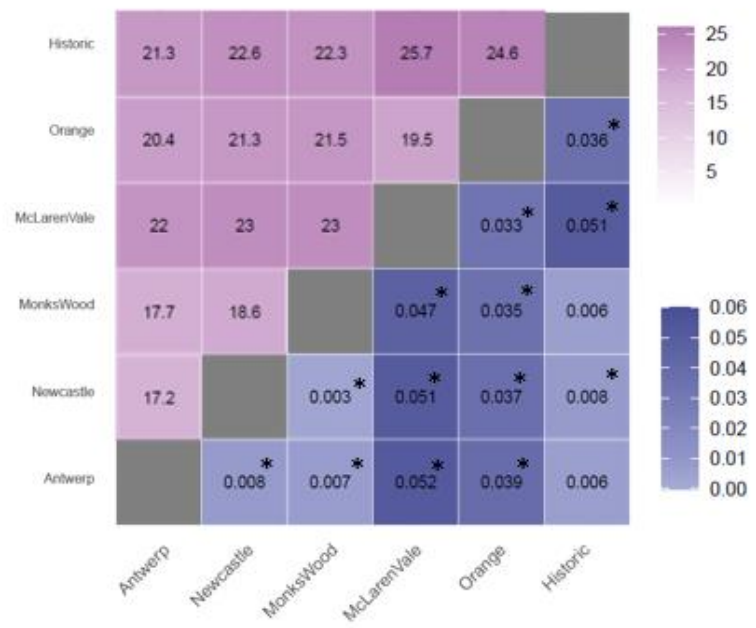

b)

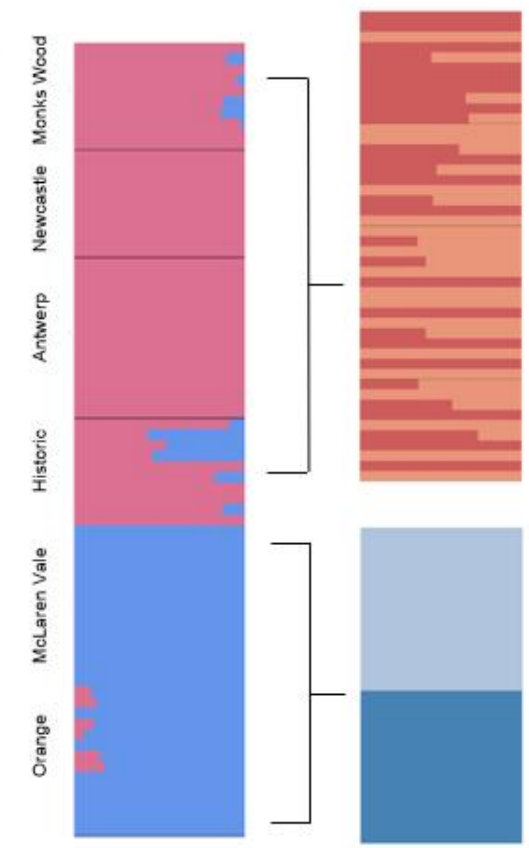

d)

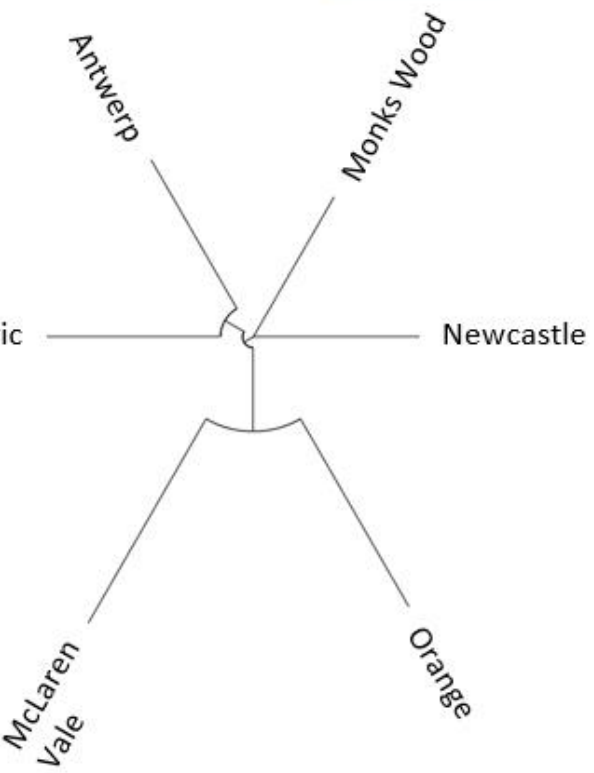

Figure 2: Population genetic analysis for contemporary and historical Sturnus vulgaris using the population genetics variant file. Panel a) denotes a PCA of the 6 sampling groups using SNPRELATE, panel $b$ ) denotes ADMIXTURE ancestry $Q$ profiles, averaged over 50 runs using CLUMPAK for all sample groups, all native range samples (contemporary and historic), contemporary native range samples, and invasive Australian samples. Panel c) displays a heatmap of pairwise analysis between each of the sample groups, with above the diagonal (purple) indicating Euclidean distance, and below the diagonal (blue) indicating pairwise $\mathrm{F}_{\mathrm{ST}}$ (asterisk * denoting a significant $\mathrm{F}_{\mathrm{ST}}$ result), and panel d) 
Table 1: Number of putative sites under selection in Sturnus vulgaris reported by the different selective scans for the pairwise comparisons of UK-HS, AU-HS, and UK-AU data set.

\begin{tabular}{|l|l|c|c|c|}
\hline Outlier group & & UK-HS & AU-HS & UK-AU \\
\hline data set SNP count & & 4997 & 5006 & 4957 \\
\hline Bayescan & FDR 0.05 & $\mathbf{8}$ & $\mathbf{1}$ & $\mathbf{1 5}$ \\
\hline & Alpha $>0.05+L D$ & $\mathbf{6 2}$ & $\mathbf{6 9}$ & $\mathbf{3 4}$ \\
& $r^{2}>0.5$ & & & \\
\hline Fst Sliding Windows & Fst $>0.5+$ SNP & $\mathbf{2 7}$ & $\mathbf{3 7}$ & $\mathbf{3 3}$ \\
& threshold & & & 71 \\
\hline Total & & 71 & 85 & \\
\hline
\end{tabular}

Table 2: SNP and gene counts of Sturnus vulgaris SNPs putatively under selection, for the five categorical grouping based on pairwise comparisons.

\begin{tabular}{|l|c|c|c|c|c|c|}
\hline Outlier SNP & & Divergent & Parallel & Putative UK & Putative & Putative UK \\
& & Selection & Selection & Selection & AU & \& AU \\
\hline SNPs & & 9 & 52 & 16 & 27 & 62 \\
\hline Coding & Total & 6 & 33 & 16 & 17 & 42 \\
\hline regions & Known & 5 & 30 & 12 & 12 & 35 \\
\hline & (BLAST+) & & & & & \\
\hline
\end{tabular}



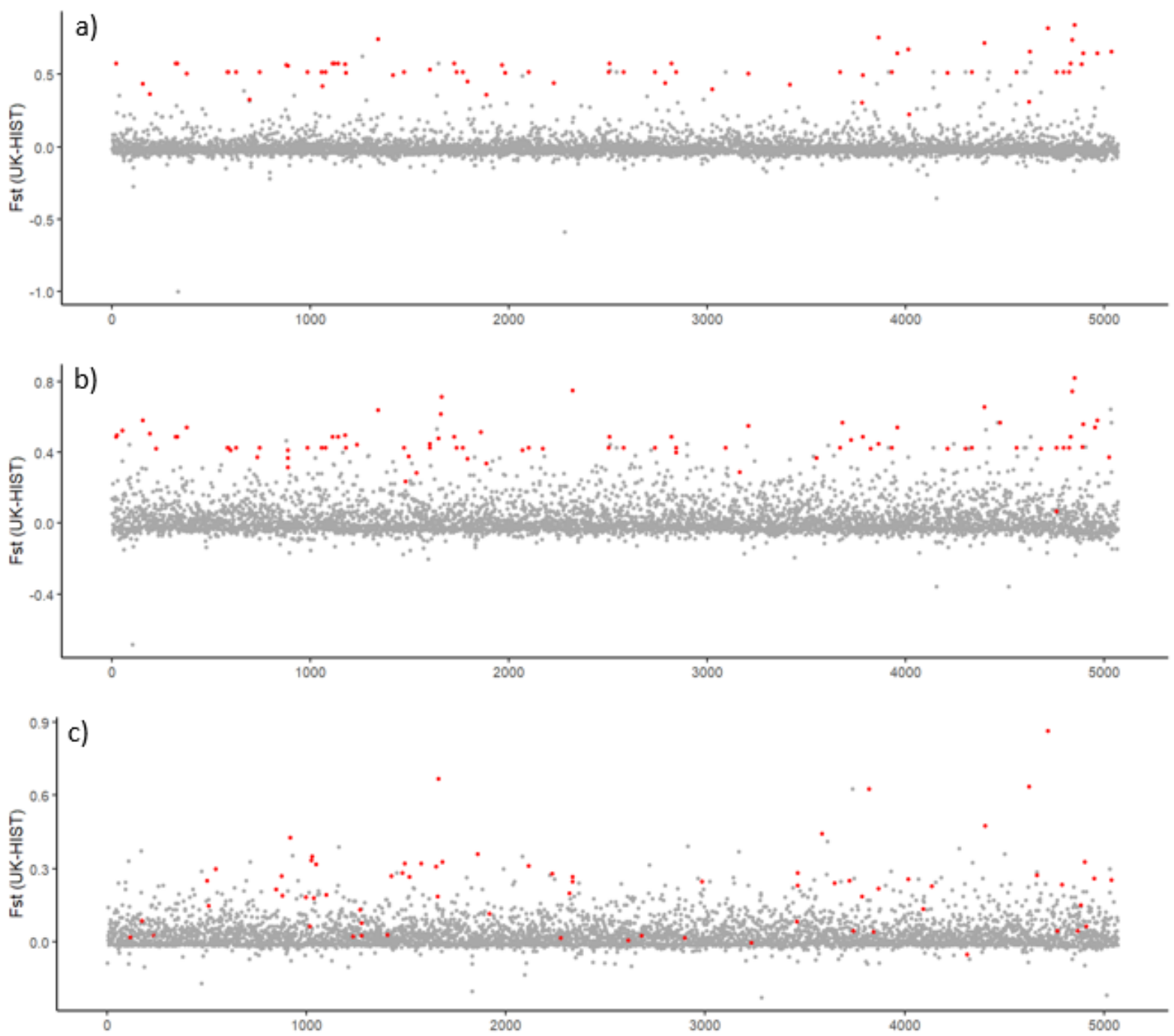

d)

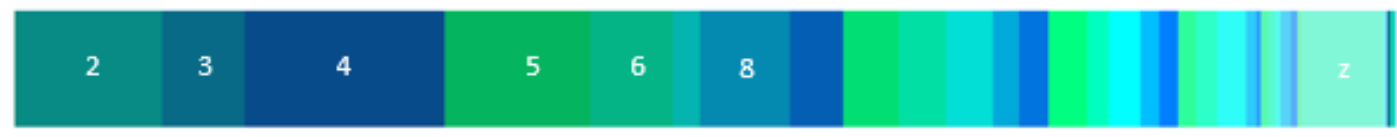

Chromosome SNP coverage

Figure 3: Mapping putatively selected loci across the Sturnus vulgaris genome. Panels a) (UK-HS samples), panel b) (AU-HS samples), and panel c) (UK-AU samples) display the weighted FST in $900 \mathrm{~kb}$ (10kb step) sliding windows as calculated by VCFTOOLS. Non-outlier SNPs are plotted in grey, while outlier SNPs are plotted in red. Panel d) depicts the major scaffolds of the $S$. vulgaris genome assembly, sized for their representation in the SNPs plotted in panels a-c). 
a)

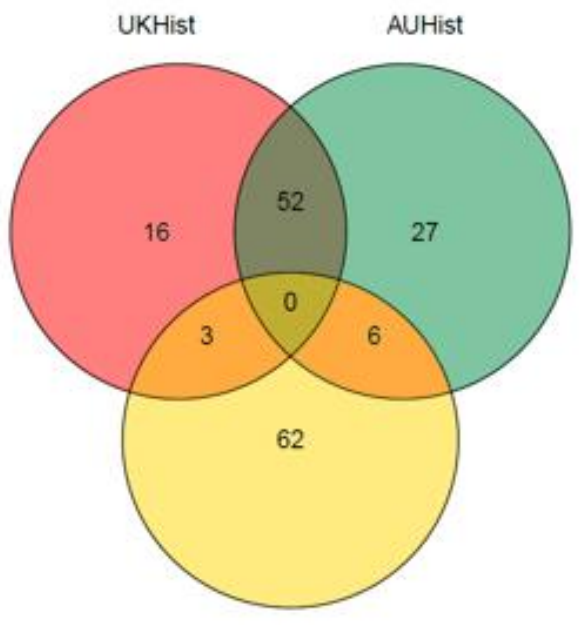

Divergent SNPs

Parallel SNPs

UK Putative Selection SNPs

AU Putative Selection SNPs

$\square \mathrm{AU}$ and UK Putative Selection SNPs

c)

b)

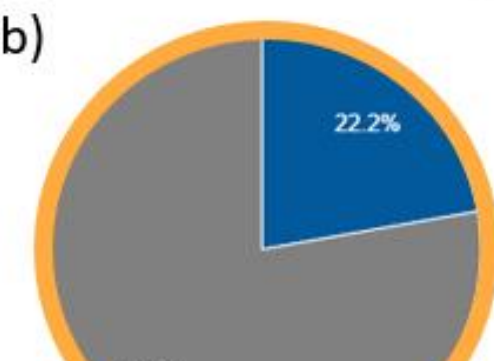

e)

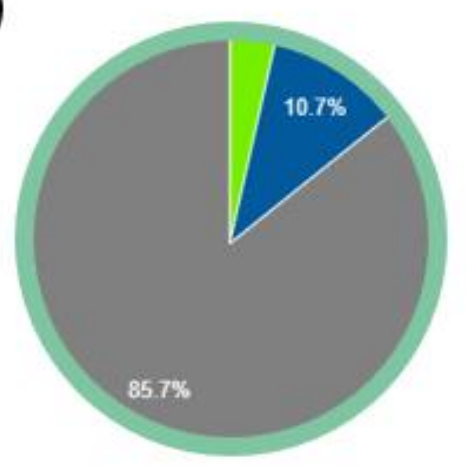

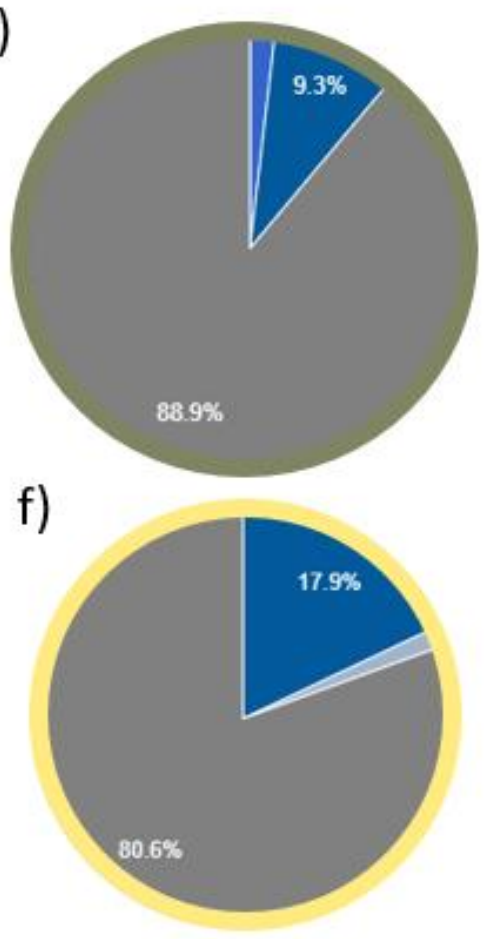

$77.8 \%$

d)

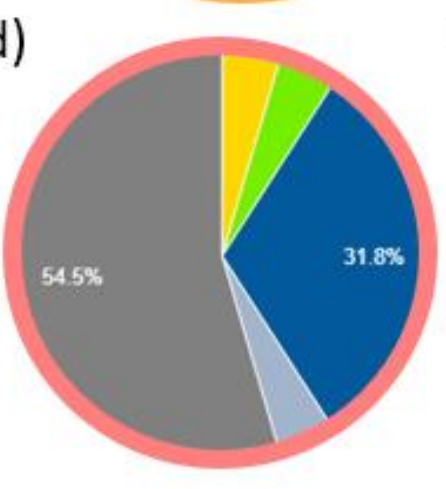

synonymous_variant

missense_variant

3_prime_UTR_variant

intron_variant

upstream_gene_variant

downstream_gene_variant

intergenic_variant

\section{)}

Figure 4: Summary of Sturnus vulgaris putative SNPs under selection, with panel a) depicting a Venn diagram of group categorisation, and the remaining figures the VARIANT EFFECT PREDICTOR (VEP) summary outputs of function variation for each of the five SNP groups of $\mathbf{b})$ SNPs under divergent selection, c) SNPs under parallel selection, d) UK SNPs under putative selection, e) AU SNPs under putative selection, and f) UK and AU SNPs under putative divergence. 
bioRxiv preprint doi: $\mathrm{https}$ //doi. org/10.1101/2021.08.22 457241; this version posted August 23,2021 The copyright holder for this preprint (which was not certified by peer review) is the author/funder, who has granted bioRxiv a license to display the preprint in perpetuity. It is made available under aCC-BY-NC-ND 4.0 International license.

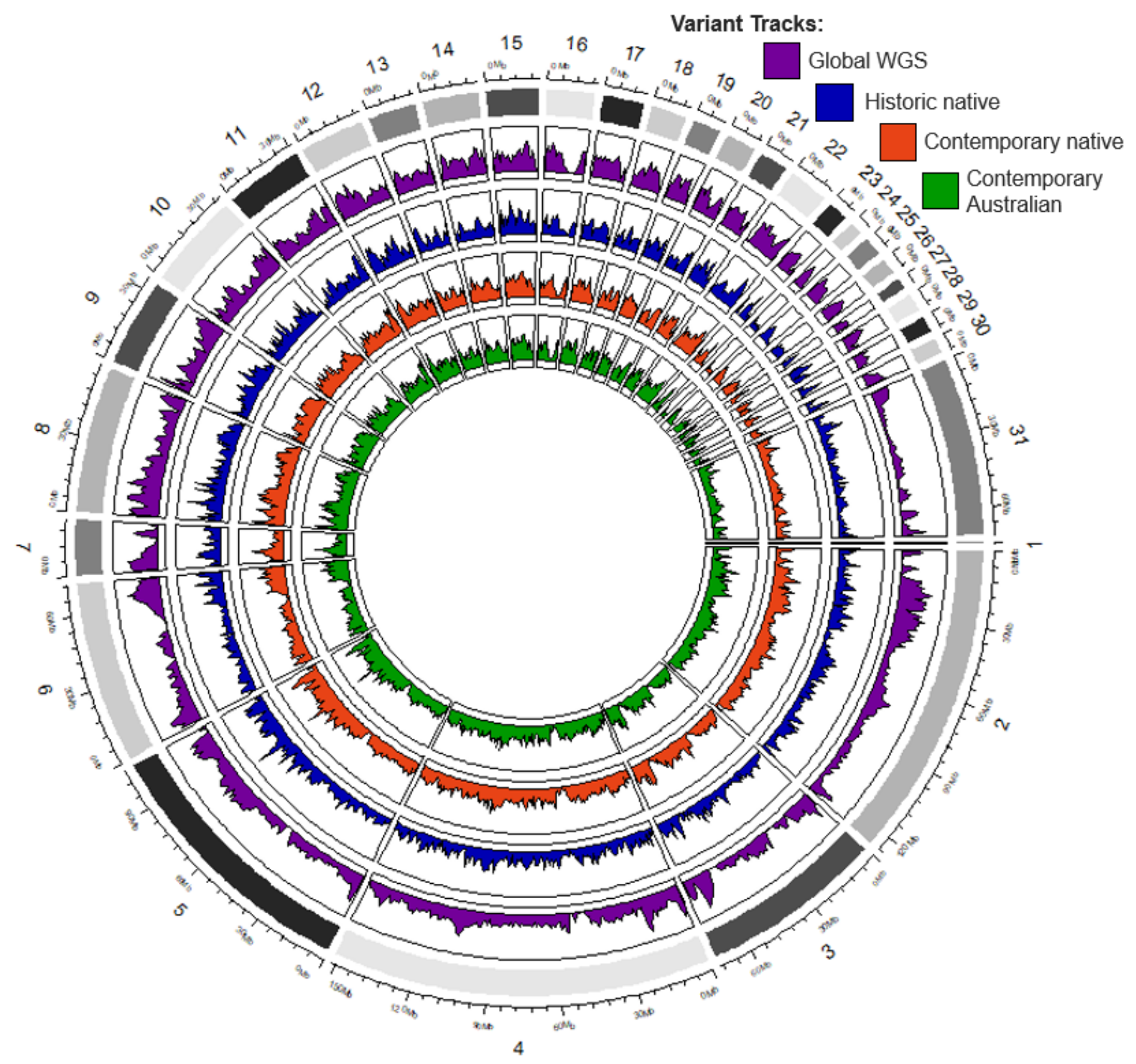

454

455

456

457

458

459

460
Figure 5: Sturnus vulgaris WGS and reduced representation sequencing SNP variant density plotted in 1,000,000 bp windows around the genome (first 31 scaffolds) for Illumina whole genome global starling variant data (purple), historical samples (blue), contemporary native range samples (red) and contemporary Australian samples (green). 
a)

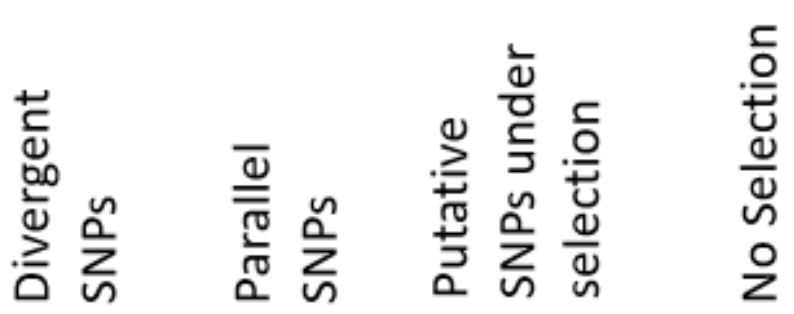

Macrochromosomes

Microchromosomes

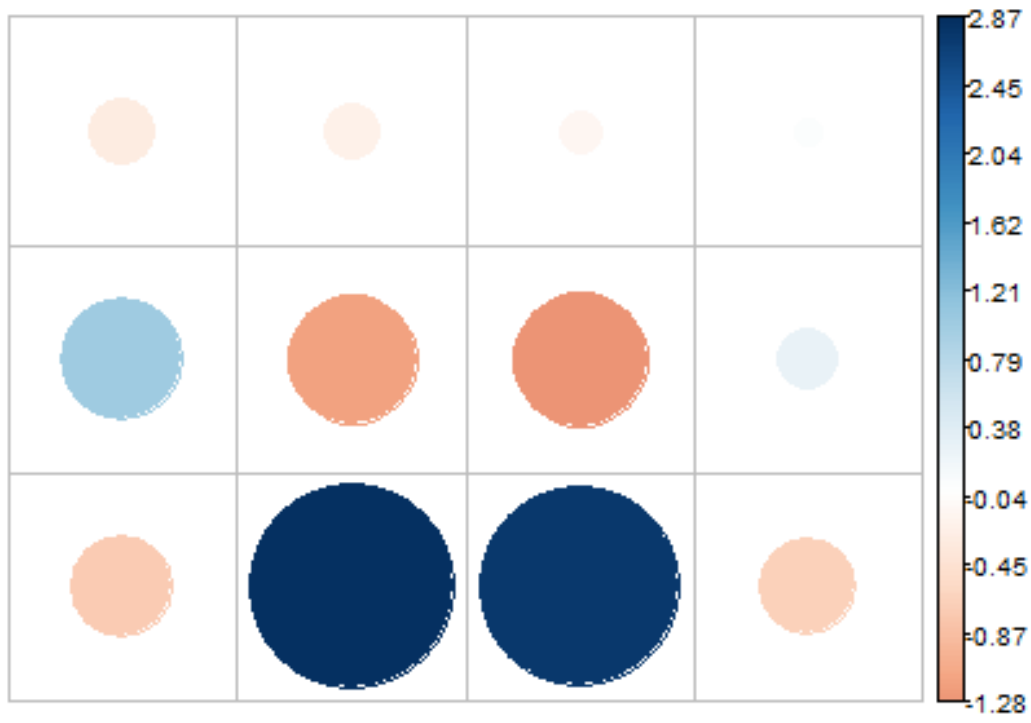

b)

Sex Chromosome

)

\section{Macrochromosomes}

Microchromosomes

Sex Chromosome

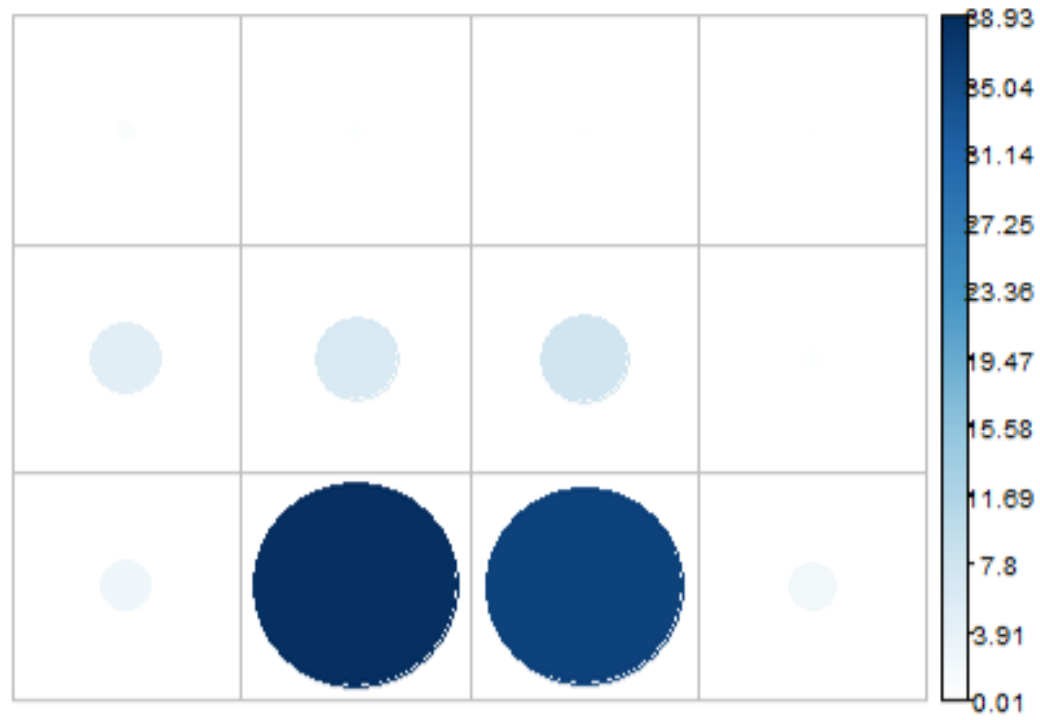

Figure 6: Test of statistical association between SNPs categorised as under selection versus the chromosome type they reside in for Sturnus vulgaris DArT-Seq, where panel a) depicts visualization of Pearson residuals, where the size of the circle is proportional to the amount of the cell contribution, positive residuals (indicating a positive correlation) are in blue, and negative residuals (indicating a negative correlation) are in orange, and panel b) depicts relative contribution of each cell to the total Chi-square score. 


\section{Discussion:}

471 This study demonstrates that S. vulgaris has not only undergone divergent selection within the

472 invasive Australian range, but that the native and invasive ranges are undergoing parallel selection, possibly in response to global environmental changes. We note that contemporary native range populations, when considering comparative numbers of divergent SNPs, have undergone a similar amount of genetic change when compared to invasive range populations, despite the latter presumably being exposed to radically different and novel selection regimes. Moreover, we identified several genes related to pollution and carbohydrate metabolism that appear to be under parallel selection in the contemporary native and invasive range samples, which may reflect global environmental changes over the last century and a half. The genes reported as divergent between the populations capture differing selection regimes driving evolution within the two contemporary populations. We also identified a bias for selection on the $Z$ chromosome in comparison to the autosomes. Importantly, this study has successfully used reduced representation sequencing of historic and contemporary specimens to examine selection in S. vulgaris within the native and Australian invasive ranges. While the success rate and quality of the historic specimen sequencing reads was less than the contemporary counterparts, the method nevertheless yielded sufficient SNP data to enable examination of population structure and description of temporal patterns of genomic change in starling populations.

\subsection{Population structure}

Very little native range $S$. vulgaris genetic data exists, hence our study provides much needed insight into the population structure and genetic variation of the UK region of the native range. We identified low levels of genetic differentiation across the native range. Some native range starlings are migratory (Feare 1984), and this large-scale dispersion undoubtedly helps to maintain genetic 
genetic resemblance to contemporary samples from the native range than those from the invasive

Australian population. The historical samples are most differentiated from their contemporary counterparts in the PCA analysis as compared to analyses of admixture, $\mathrm{F}_{S T}$, genetic distance and phylogeny. Interestingly, admixture analysis depicts historic samples as a mixture of contemporary native and invasive genotypes, though with the UK cluster appearing more predominantly. Genotype substructure is therefore indicative of shifting starling genetics over the last 160 years within the native range. interesting that the only contemporary UK population that had a statistically different $\mathrm{F}_{\text {ST }}$ measure statistically different, they are not biologically important as they represent a negligible difference in allelic variance that may have been affected by study specifics such as sampling scheme. The different population genetics analyses conducted reported that either Monks Wood or Antwerp bears the strongest resemblance to the historic samples, the different results likely a result of genetic subclusters (Stuart \& Cardilini et al. 2021a), and further reinforces the idea that there were

512 slight but distinct genetic differences in the disparate introductions of founding individuals.

513 Comparing the contemporary Australian sample sites to GBS sequencing in the same regions (Stuart

514 \& Cardilini et al. 2021a), suggests that the sample sizes in this study were sufficient to be

515 representative of the genetic variation at sampling locations, and that between sample site genetic divergence is higher within this invasive when compared to the native UK over a comparable 


\subsection{Genomic divergence}

Of the three outlier selection methods used, the default BAYESCAN approach identified the

fewest sites. This was likely due to the small sample sizes lending less statistical power to this stricter

analysis, meaning the program was unable to pick up the low signals of selection in these recently

using BAYESCAN approaches did not always align with $\mathrm{F}_{\mathrm{ST}}$ peaks from what, particularly in the UK-AU

data set (Fig 3). These flagged outliers with lower SNP site F $_{S T}$ were made up proportionally of the

SNPs flagged using the BAYESCAN default and alpha+LD approach (with the Fst outlier method inherently selecting against lower SNP site F $_{\text {ST }}$. Because the UK-AU data set contained a larger number of individuals than the pairwise comparisons that incorporated the historical data, it is reasonable to conclude that these are likely legitimate but weaker outliers that were able to be identified due to increased statistical power. With smaller historical sample sizes, detection will likely be biased towards highly diverged SNPs, which have high F $_{S T}$ values. selection (62 SNPs), in comparison to putative UK selection (16) and putative AU selection (27). This is likely because lower statistical power of smaller historic sample sizes failed to flag some sites under relatively weaker selection in the UK-HS and AU-HS pairwise analysis. These aforementioned SNPS were the ones that were only flagged in one of the pairwise comparisons, and therefore were not 536 categorised as either parallel or divergent. Interestingly, for the SNPs that were categorised, we see approximately five times the number of SNPs under parallel selection (52 SNPs) in comparison to divergent SNPs (9 SNPs). Across these five SNP selection lists, we identified few variants that were predicted to alter coding potentials within a gene. Other than intergenic variants, intron variants made up the highest proportion of SNPs. Despite not being transcribed gene regions, introns may 
2017). We also observed a bias towards the larger sex chromosome (Z) in terms of SNPs that were categorised as being under parallel selection (and also within the UK, AU, and UK and AU SNPs under putative selection). It is possible that this may have arisen due to biases in aspects of the data (e.g. sequencing method, SNP variant calling pipeline). However, we found no differences in major allele frequency or allele variant numbers (factors which may impact a SNP site's ability to be flagged in the outlier analysis methods used) across SNPs categorised as either under selection or not, across sex chromosomes and autosomes. The conclusion does align with theory that suggests that sex chromosomes are capable of playing a disproportionate role in evolutionary divergence due to their haploid nature in one sex (e.g. the 'faster-X effect'; Meisel and Connallon 2013), and may be one of the first steps towards speciation (Oyler-McCance et al. 2015; Wilson Sayres 2018).

SNPs under putative parallel selection were mostly seen to have higher allelic diversity in the historical samples, with both contemporary populations becoming fixed (or nearly so) for the same allelic variant (supplementary materials, Table S4). This may be indicative of beneficial/nondeleterious alleles shifting towards fixation within contemporary populations, linkage with a nearby variant, or be a result of random processes such as drift (though this latter explanation would be quite unlikely for parallel processes). This over-representation of fixation in contemporary populations is unsurprising, because parallel evolution is more likely to have been based on standing genetic variation in the ancestral population (variants that were already there) than on the same novel variants arising independently in the two descendant populations. We do see apparent fixation

561 in historical populations and more mixed allele frequencies within contemporary populations, in a 562 small number of cases within the parallel SNP data. Divergence within the AU population may be a 563 result of invasion bottleneck processes biasing allelic variation for or against rare variants (depending on their representation in the translocated individuals). However, given that several hundred individuals were introduced to Australia, it would be very unlikely for common allelic variants to be 
lost through such random processes and more likely that the observed allele frequencies are a result of factors post-introduction (e.g. selection against non-local maladaptation, drift).

\subsection{Genes undergoing putative adaptive selection}

Of the genes that have possibly undergone parallel selection in both the native and invasive range,

we see a number of these genes which have been previously associated with responses to various

571 pollutants. Exposure to HT-2, a grain mycotoxin, has been shown to downregulate nidogen 2 (NID2)

572 expression in bovines (Li et al. 2020a). Starling interaction with livestock is well documented, and

573 indeed is a key motivating factor of their management in agricultural areas (Linz et al. 2007). With

574 increasing livestock numbers, grazing areas, and feed grain use (Spragg 2018), it is not unreasonable

575 for birds that opportunistically feed from grain stocks to face increased exposure to grain-borne

576 toxins and pollutants. Another pollutant, $\operatorname{TCDD}(2,3,7,8$-tetrachlorodibenzodioxin), is persistent and

577 globally used, and has been documented to alter cell cycle progression and proliferation, mediated

578 through the gene Son of sevenless homolog 1 (SOS1) (Pierre et al. 2011). Dioxins have a high

579 solubility in lipids, and coupled with a chemical half-life of 9-100 years (depending on substrate),

580 bioaccumulation of these toxins in animals and plants is a well-documented and significant health

581 concern (National Dioxins Program (Australia) et al. 2004). Over the last 160 years, increased

582 modernisation globally has resulted in organisms being exposed to increased levels of pollutants.

583 Even trace amounts of compounds may be detrimental to some organisms (Kozlov et al. 2009; Bucci

584 et al. 2020), and starling eggs have been demonstrated to accumulate polluting organic compounds

585 (Eens et al. 2013). Global bird numbers have been declining, across both rare and common species

586 (Gross 2015; Li et al. 2020b), with particular toxins such as neonicotinoid from insecticides being

587 documented to play a major role in avian insectivore decline (Hallmann et al. 2014). Understanding

588 which pollutants species are coping with and which are not may be important for understanding 
species and range persistence patterns and may shed light onto the various factors influencing native range starling declines.

Two genes related to carbohydrate metabolic processing were flagged as under parallel selection across the native and invasive range: sucrase-isomaltase, intestinal (SI) and insulin (INS). Gene $\mathrm{Sl}$ encodes for an intestinal tract enzyme involved in breaking down sucrose (obtained from e.g. fruit) and maltose (e.g. grain). Further, INS is a pivotal hormone that regulates the metabolism of carbohydrates and lipids. Starlings naturally feed on invertebrates, however shifts towards intensive animal farming and agricultural land expansion globally (Macdonald \& McBride 2009) have likely caused increased opportunistic feeding on sucrose and maltose via livestock feed (Linz et al. 2007). While evidence suggests that starlings possess limited sucrose processing ability (Martínez del Rio 1990), alterations of allele frequencies within these two genes in both AU and UK populations has implications for selection on metabolic processes that may interact with human supplied food sources, either in the face of the increasing availability of human-supplied food or due to declines in invertebrate numbers (Hallmann et al. 2017).

Within the genes that are possibly undergoing divergent selection across the native and invasive range, gene ontology analysis indicates that the biological processes of signal transduction and ion transport are over-represented. Only one gene was reported as undergoing divergence within the native range: Ankyrin repeat and KH domain-containing protein 1 (ANKHD1) (supplementary materials, Table S4, Table S5). ANKHD1 plays an important role in cell cycle progression and proliferation, and has been associated with cancers in humans and model organisms (Dhyani et al. 2012; Machado-Neto et al. 2014). Four genes, estrogen related receptor gamma (Esrrg), Glutamate lonotropic Receptor Kainate Type Subunit 2 (GRIK2), Rho GTPase-activating protein 10 (ARHGAP10), and Voltage-dependent calcium channel subunit alpha-2/delta-3 (Cacna2d3) are reported as divergent in the invasive AU range (supplementary materials, Table S4, Table S5). Of 
particular interest, the gene Esrrg is important for regulating metabolism and energy production in cells, and has been identified as a possible adaptive feature of the desert environment in dromedary camels (Bahbahani et al. 2019). It is feasible that all these biological functions would assist starlings as they established and colonised the novel and arid environment of Australia. Doubtless, comparisons to starlings sampled from other arid invasive regions (inland North America and South Africa), as well as the northern African native range edges would provide insight into arid adaptation variation in this species globally. signalling-mediated neurogenesis, and may be a regulatory gene related to flightlessness in Galapagos cormorants (Berger \& Bejerano 2017). Lastly GRIK2, involved in the functional molecular organisation of the avian cerebrum (Jarvis et al. 2013), and CACNA2D3 involved in neurexin-mediated retrograde signaling (Tong et al. 2017) and may play an important role in pathways for learned vocalisation (Wada et al. 2004; Friedrich et al. 2019). Although the biological functions associated with these genes are broad, this result provides candidate genes for future studies investigating altered dispersion physiology and vocalisation. putatively under selection across the starling's global ranges. There are undoubtedly more sites of selection, either only identified in one of the outliers pairwise data sets (and so not categorised as parallel or divergent), in linkage with the identified SNPs (Brodie et al. 2016), or not sequenced at all using this reduced representation approach. However, these variants and genes serve as a shortlist of suitable targets for future gene expression analysis, or as the basis of further hypothesis on global avian selection pressures. 


\subsection{Historic sample sequencing}

638

639

640

641

The success rate of historic sample sequencing was around $70 \%$, with no correlation between sample properties and sequencing success. Patterns of density of variants across the genome identified using DArTseq appeared to follow similar patterns to the high-quality variant density data set provided by the whole genome comparison (Fig. 5), although historic samples did report a patchier variant distribution. However, prior simulations using data from historical samples reported that though historic samples contain significantly more missing data when compared to fresh tissue samples, the level of genotypic error had a minimal effect on population structure inference (Ewart et al. 2019). In terms of sequence data processing, the BWA Aln-Stacks and BWA Mem-Stacks pipelines performed similarly, with bowtie-GATK performing comparatively much worse. This large difference may be due to the large amounts of missing data (Catchen et al. 2013), but are different from the relative performance previously reported for these two variant calling pipelines (Wright et al. 2019), suggesting that variant calling success is very data set dependent.

\subsection{Future directions}

Greater coverage of native range starling genetics is a vital future step for evolutionary genomic studies on this species. Improved native range genetic data will both help us better understand population structure and allelic shifts in the invasive ranges, and also shed light on native range dispersion dynamics. Further, the success of the museum sample sequencing demonstrated here gives hope that when largescale genomic studies are conducted in the native range, historical samples will be able to provide crucial background information regarding genetic diversity prior to the significant population declines.

Analyses of a greater number of historical samples will also aid in the categorisation of adaptive SNPs, as this will reduce possible effects of random sampling bias and capture more rare alleles. The sequencing failure rate of this study is comparable to another study using similarly aged 
museum skins (Ewart et al. 2019), suggesting that future projects seeking to use museum samples might expect similar failure rates (30-40\%) and adjust their sampling design accordingly. Finally, similar analyses may be conducted between the historic UK and the well-studied contemporary North American starlings (as this population has a similar introduction time, range size, and environmental variation: Bodt et al. 2020; Hofmeister et al. 2021b), and then further extended upon through comparisons to other invasive populations in New Zealand, South Africa, and South America. Comparisons to parallel invasive populations will provide an invaluable opportunity to contrast concurrent species invasion and selection across multiple different continents, allowing for the discovery of broad evolutionary pattern in this invasive species.

\section{Conclusions:}

Overall, this study demonstrates that the combination of native, invasive, and historical genetic data can lead to a more thorough understanding of global species shifts during the Anthropocene. We use genetic sequencing of museum specimens to identify putatively adaptive genetic changes through reduced representation sequencing and outlier SNP identification analysis. We have described evidence of parallel and divergent evolution in native and invasive starlings since the mid-19 Century. Finally, we identify an apparent bias towards putatively adaptive SNPs on the Z chromosome, suggesting that the major sex chromosome may play an overly proportionate role in rapid evolution within this species.

\section{Acknowledgements:}

Thank you to Hein Van Grouw at Tring NHM for assistance with sourcing historical starling specimens. LAR was supported by a Scientia Fellowship from UNSW.

\section{Author Contributions}

Project conception: KCS, LAR 
Data Analysis: KCS

Manuscript Writing: KCS

688

Manuscript Editing: All authors

689

690

Data Accessibility:

691 The data have been deposited with links to BioProject accession number XXXXX in the NCBI

692 BioProject database (https://www.ncbi.nlm.nih.gov/bioproject/). Any scripts or metadata not

693 covered by the above will be available on GitHub.

694

695

696

697

698

699

700

701

702

703

704

705

706

707

708 


\section{REFERENCES:}

Al-Breiki RD, Kjeldsen SR, Afzal H, Al Hinai MS, Zenger KR, Jerry DR, Al-Abri MA \& Delghandi M 2018 Genome-wide SNP analyses reveal high gene flow and signatures of local adaptation among the scalloped spiny lobster (Panulirus homarus) along the Omani coastline. BMC Genomics 19 690. (doi:10.1186/s12864-018-5044-8)

Backström N, Forstmeier W, Schielzeth H, Mellenius H, Nam K, Bolund E, Webster MT, Öst T, Schneider M, Kempenaers B et al. 2010 The recombination landscape of the zebra finch Taeniopygia guttata genome. Genome Research 20 485-495. (doi:10.1101/gr.101410.109)

Bahbahani H, Musa HH, Wragg D, Shuiep ES, Almathen F \& Hanotte O 2019 Genome Diversity and Signatures of Selection for Production and Performance Traits in Dromedary Camels. Frontiers in Genetics 10. (doi:10.3389/fgene.2019.00893)

Berger MJ \& Bejerano G 2017 Comment on "A genetic signature of the evolution of loss of flight in the Galapagos cormorant". BioRxiv 181826. (doi:10.1101/181826)

Bishop P 2011 Bell frog populations in New Zealand - good news or bad news? Australian Zoologist 34 408-413. (doi:10.7882/AZ.2008.018)

Bodt LH, Rollins LA \& Zichello J 2020 Genetic Diversity of the European Starling (Sturnus vulgaris) Compared Across Three Invasive Ranges.

Brodie A, Azaria JR \& Ofran Y 2016 How far from the SNP may the causative genes be? Nucleic Acids Research 44 6046-6054. (doi:10.1093/nar/gkw500)

Bucci K, Tulio M \& Rochman CM 2020 What is known and unknown about the effects of plastic pollution: A meta-analysis and systematic review. Ecological Applications 30 e02044. (doi:10.1002/eap.2044)

Callaway RM \& Ridenour WM 2004 Novel weapons: invasive success and the evolution of increased competitive ability. Frontiers in Ecology and the Environment 2 436-443. (doi:https://doi.org/10.1890/1540-9295(2004)002[0436:NWISAT]2.0.CO;2)

Catchen J, Hohenlohe PA, Bassham S, Amores A \& Cresko WA 2013 Stacks: an analysis tool set for population genomics. Molecular Ecology 22 3124-3140. (doi:10.1111/mec.12354)

Dainat J 2020 AGAT: Another Gff Analysis Toolkit to Handle Annotations in Any GTF/GFF Format. Zenodo.

Danecek P, Auton A, Abecasis G, Albers CA, Banks E, DePristo MA, Handsaker RE, Lunter G, Marth GT, Sherry ST et al. 2011 The variant call format and VCFtools. Bioinformatics 27 2156-2158. (doi:10.1093/bioinformatics/btr330)

Delibes-Mateos M, Ferreras P \& Villafuerte R 2009 European rabbit population trends and associated factors: a review of the situation in the Iberian Peninsula. Mammal Review 39 124-140. (doi:https://doi.org/10.1111/j.1365-2907.2009.00140.x) 
Deng HW, Chen WM \& Recker RR 2001 Population admixture: detection by Hardy-Weinberg test and its quantitative effects on linkage-disequilibrium methods for localizing genes underlying complex traits. Genetics 157 885-897.

Dhyani A, Duarte ASS, Machado-Neto JA, Favaro P, Ortega MM \& Olalla Saad ST 2012 ANKHD1 regulates cell cycle progression and proliferation in multiple myeloma cells. FEBS Letters 586 4311-4318. (doi:10.1016/j.febslet.2012.10.037)

Dukes JS \& Mooney HA 1999 Does global change increase the success of biological invaders? Trends in Ecology \& Evolution 14 135-139. (doi:10.1016/\$0169-5347(98)01554-7)

Eens M, Jaspers VLB, Van den Steen E, Bateson M, Carere C, Clergeau P, Costantini D, Dolenec Z, Elliott JE, Flux J et al. 2013 Can starling eggs be useful as a biomonitoring tool to study organohalogenated contaminants on a worldwide scale? Environment International 51 141149. (doi:10.1016/j.envint.2012.11.003)

Erfmeier A \& Bruelheide H 2010 Invasibility or invasiveness? Effects of habitat, genotype, and their interaction on invasive Rhododendron ponticum populations. Biological Invasions 12 657676. (doi:10.1007/s10530-009-9472-x)

Ewart KM, Johnson RN, Ogden R, Joseph L, Frankham GJ \& Lo N 2019 Museum specimens provide reliable SNP data for population genomic analysis of a widely distributed but threatened cockatoo species. Molecular Ecology Resources 19 1578-1592. (doi:10.1111/17550998.13082)

Feare CJ 1984 The Starling. Shire.

Foll M \& Gaggiotti O 2008 A Genome-Scan Method to Identify Selected Loci Appropriate for Both Dominant and Codominant Markers: A Bayesian Perspective. Genetics 180 977-993. (doi:10.1534/genetics.108.092221)

Freeman SN, Robinson RA, Clark JA, Griffin BM \& Adams SY 2007 Changing demography and population decline in the Common Starling Sturnus vulgaris: a multisite approach to Integrated Population Monitoring. Ibis 149 587-596. (doi:10.1111/j.1474919X.2007.00684.x)

Friedrich SR, Lovell PV, Kaser TM \& Mello CV 2019 Exploring the molecular basis of neuronal excitability in a vocal learner. BMC Genomics 20 629. (doi:10.1186/s12864-019-5871-2)

Gloria-Soria A, Dunn WA, Telleria EL, Evans BR, Okedi L, Echodu R, Warren WC, Montague MJ, Aksoy $S$ \& Caccone A 2016 Patterns of Genome-Wide Variation in Glossina fuscipes fuscipes Tsetse Flies from Uganda. G3 (Bethesda, Md.) 6 1573-1584. (doi:10.1534/g3.116.027235)

Gross M 2015 Europe's bird populations in decline. Current Biology 25 R483-R485. (doi:10.1016/j.cub.2015.05.057)

Gruber B, Unmack PJ, Berry OF \& Georges A 2018 dartr: An r package to facilitate analysis of SNP data generated from reduced representation genome sequencing. Molecular Ecology Resources 18 691-699. (doi:10.1111/1755-0998.12745) 
Hallmann CA, Foppen RPB, van Turnhout CAM, de Kroon H \& Jongejans E 2014 Declines in insectivorous birds are associated with high neonicotinoid concentrations. Nature 511 341343. (doi:10.1038/nature13531)

Hallmann CA, Sorg M, Jongejans E, Siepel H, Hofland N, Schwan H, Stenmans W, Müller A, Sumser H, Hörren T et al. 2017 More than 75 percent decline over 27 years in total flying insect biomass in protected areas. PLOS ONE 12 e0185809. (doi:10.1371/journal.pone.0185809)

Heldbjerg H, Fox AD, Levin G \& Nyegaard T 2016 The decline of the Starling Sturnus vulgaris in Denmark is related to changes in grassland extent and intensity of cattle grazing. Agriculture, Ecosystems \& Environment 230 24-31. (doi:10.1016/j.agee.2016.05.025)

Hellmann JJ, Byers JE, Bierwagen BG \& Dukes JS 2008 Five Potential Consequences of Climate Change for Invasive Species. Conservation Biology 22 534-543. (doi:https://doi.org/10.1111/j.15231739.2008.00951.x)

Higgins PJ, Peter JM \& Cowling SJ 2006 Handbook of Australian, New Zealand \& Antarctic birds. Volume 7, Boatbill to starlings. Melbourne : Oxford University Press.

Hoffmann BD \& Broadhurst LM 2016 The economic cost of managing invasive species in Australia. NeoBiota 31 1-18. (doi:10.3897/neobiota.31.6960)

Hofmeister NR, Stuart K, Warren WC, Werner SJ, Bateson M, Ball GF, Buchanan KL, Burt DW, Cardilini APA, Cassey $P$ et al. 2021a Concurrent invasions by European starlings (Sturnus vulgaris) suggest selection on shared genomic regions even after genetic bottlenecks. BioRxiv 2021.05.19.442026. (doi:10.1101/2021.05.19.442026)

Hofmeister NR, Werner SJ \& Lovette IJ 2021b Environmental correlates of genetic variation in the invasive European starling in North America. Molecular Ecology 30 1251-1263. (doi:10.1111/mec.15806)

Hulme PE 2009 Trade, transport and trouble: managing invasive species pathways in an era of globalization. Journal of Applied Ecology 46 10-18. (doi:10.1111/j.1365-2664.2008.01600.x)

Jarvis ED, Yu J, Rivas MV, Horita H, Feenders G, Whitney O, Jarvis SC, Jarvis ER, Kubikova L, Puck AEP et al. 2013 Global view of the functional molecular organization of the avian cerebrum: Mirror images and functional columns. Journal of Comparative Neurology 521 3614-3665. (doi:https://doi.org/10.1002/cne.23404)

Kilian A, Wenzl P, Huttner E, Carling J, Xia L, Blois H, Caig V, Heller-Uszynska K, Jaccoud D, Hopper C et al. 2012 Diversity arrays technology: a generic genome profiling technology on open platforms. Methods in Molecular Biology (Clifton, N.J.) 888 67-89. (doi:10.1007/978-1-61779870-2_5)

Kopelman NM, Mayzel J, Jakobsson M, Rosenberg NA \& Mayrose I 2015 Clumpak: a program for identifying clustering modes and packaging population structure inferences across $\mathrm{K}$. Molecular Ecology Resources 15 1179-1191. (doi:10.1111/1755-0998.12387)

Kozlov M, Zvereva E \& Zverev V 2009 Impacts of Point Polluters on Terrestrial Biota: Comparative Analysis of 18 Contaminated Areas. Springer Science \& Business Media. 
Li H 2013 Aligning sequence reads, clone sequences and assembly contigs with BWA-MEM. ArXiv:1303.3997 [q-Bio].

Li H \& Durbin R 2009 Fast and accurate short read alignment with Burrows-Wheeler transform. Bioinformatics 25 1754-1760. (doi:10.1093/bioinformatics/btp324)

Li H, Handsaker B, Wysoker A, Fennell T, Ruan J, Homer N, Marth G, Abecasis G, Durbin R, \& 1000 Genome Project Data Processing Subgroup 2009 The Sequence Alignment/Map format and SAMtools. Bioinformatics (Oxford, England) 25 2078-2079. (doi:10.1093/bioinformatics/btp352)

Li L, Yang M, Li C, Yang F \& Wang G 2020a Understanding the Toxin Effects of $\beta$-Zearalenol and HT-2 on Bovine Granulosa Cells Using iTRAQ-Based Proteomics. Animals : An Open Access Journal from MDPI 10. (doi:10.3390/ani10010130)

Li Y, Miao R \& Khanna M 2020b Neonicotinoids and decline in bird biodiversity in the United States. Nature Sustainability 3 1027-1035. (doi:10.1038/s41893-020-0582-x)

Lindenbaum P 2015 JVarkit: Java-Based Utilities for Bioinformatics. figshare.

Linz GM, Homan HJ, Gaulker SM, Penry LB \& Bleier WJ 2007 European starlings: A review of an invasive species with far-reaching impacts. Managing Vertebrate Invasive Species 24.

Liu D \& Trumble JT 2007 Comparative fitness of invasive and native populations of the potato psyllid (Bactericera cockerelli). Entomologia Experimentalis et Applicata 123 35-42. (doi:https://doi.org/10.1111/j.1570-7458.2007.00521.x)

Long JL 1981 Introduced Birds of the World: The Worldwide History, Distribution, and Influence of Birds Introduced to New Environments. Terrey Hills, Sydney, NSW: Universe Books.

Lopez L, Turner KG, Bellis ES \& Lasky JR 2020 Genomics of natural history collections for understanding evolution in the wild. Molecular Ecology Resources 20 1153-1160. (doi:https://doi.org/10.1111/1755-0998.13245)

Macdonald J \& McBride W 2009 The Transformation of U.S. Livestock Agriculture: Scale, Efficiency, and Risks. United States Department of Agriculture, Economic Research Service, Economic Information Bulletin. (doi:10.2139/ssrn.1354028)

Machado-Neto JA, Lazarini M, Favaro P, Franchi GC, Nowill AE, Saad STO \& Traina F 2014 ANKHD1, a novel component of the Hippo signaling pathway, promotes YAP1 activation and cell cycle progression in prostate cancer cells. Experimental Cell Research 324 137-145. (doi:10.1016/j.yexcr.2014.04.004)

Martínez del Rio C 1990 Dietary, Phylogenetic, and Ecological Correlates of Intestinal Sucrase and Maltase Activity in Birds. Physiological Zoology 63 987-1011.

McLaren W, Gil L, Hunt SE, Riat HS, Ritchie GRS, Thormann A, Flicek P \& Cunningham F 2016 The Ensembl Variant Effect Predictor. Genome Biology 17 122. (doi:10.1186/s13059-016-0974-4)

Meisel RP \& Connallon T 2013 The faster-X effect: integrating theory and data. Trends in Genetics 29 537-544. (doi:10.1016/j.tig.2013.05.009) 
National Dioxins Program (Australia), Gatehouse R, Australia, Department of the Environment and Heritage, \& Chemical Assessment Section 2004 Ecological Risk Assessment of Dioxins in Australia. Canberra, A.C.T.: Australian Government Department of the Environment and Heritage.

Oyler-McCance SJ, Cornman RS, Jones KL \& Fike JA 2015 Z chromosome divergence, polymorphism and relative effective population size in a genus of lekking birds. Heredity 115 452-459. (doi:10.1038/hdy.2015.46)

Pierre S, Bats A-S, Chevallier A, Bui L-C, Ambolet-Camoit A, Garlatti M, Aggerbeck M, Barouki R \& Coumoul X 2011 Induction of the Ras activator Son of Sevenless 1 by environmental pollutants mediates their effects on cellular proliferation. Biochemical Pharmacology 81304 313. (doi:10.1016/j.bcp.2010.10.003)

Prentis PJ, Wilson JRU, Dormontt EE, Richardson DM \& Lowe AJ 2008 Adaptive evolution in invasive species. Trends in Plant Science 13 288-294. (doi:10.1016/j.tplants.2008.03.004)

R Core Team 2017 R: A Language and Environment for Statistical Computing. Vienna, Austria: R Foundation for Statistical Computing.

Robinson KM, Hawkins AS, Santana-Cruz I, Adkins RS, Shetty AC, Nagaraj S, Sadzewicz L, Tallon LJ, Rasko DA, Fraser CM et al. 2017 Aligner optimization increases accuracy and decreases compute times in multi-species sequence data. Microbial Genomics 3. (doi:10.1099/mgen.0.000122)

Rochette NC \& Catchen JM 2017 Deriving genotypes from RAD-seq short-read data using Stacks. Nature Protocols 12 2640-2659. (doi:10.1038/nprot.2017.123)

Rochette NC, Rivera-Colón AG \& Catchen JM 2019 Stacks 2: Analytical methods for paired-end sequencing improve RADseq-based population genomics. Molecular Ecology 28 4737-4754. (doi:10.1111/mec.15253)

Rogers DL, Matheson AC, Vargas-Hernández JJ \& Guerra-Santos JJ 2006 Genetic Conservation of Insular Populations of Monterey Pine (Pinus Radiata D. Don). Biodiversity \& Conservation 15 779-798. (doi:10.1007/s10531-004-1066-4)

Rollins LA, Woolnough AP, Wilton AN, Sinclair R \& Sherwin WB 2009 Invasive species can't cover their tracks: using microsatellites to assist management of starling (Sturnus vulgaris) populations in Western Australia. Molecular Ecology 18 1560-1573. (doi:10.1111/j.1365294X.2009.04132.x)

Rollins LA, Woolnough AP, Sinclair R, Mooney NJ \& Sherwin WB 2011 Mitochondrial DNA offers unique insights into invasion history of the common starling. Molecular Ecology 20 23072317. (doi:10.1111/j.1365-294X.2011.05101.x)

Selechnik D, Richardson MF, Hess MK, Hess AS, Dodds KG, Martin M, Chan TC, Cardilini APA, Sherman $\mathrm{CDH}$, Shine R et al. 2020 Inherent population structure determines the importance of filtering parameters for reduced representation sequencing analyses. BioRxiv 2020.11.14.383240. (doi:10.1101/2020.11.14.383240) 
Shaul O 2017 How introns enhance gene expression. The International Journal of Biochemistry \& Cell Biology 91 145-155. (doi:10.1016/j.biocel.2017.06.016)

Siepielski AM, Morrissey MB, Buoro M, Carlson SM, Caruso CM, Clegg SM, Coulson T, DiBattista J, Gotanda KM, Francis CD et al. 2017 Precipitation drives global variation in natural selection. Science 355 959-962. (doi:10.1126/science.aag2773)

Slatkin M 2008 Linkage disequilibrium - understanding the evolutionary past and mapping the medical future. Nature Reviews. Genetics 9 477-485. (doi:10.1038/nrg2361)

Sokolova IM \& Lannig G 2008 Interactive effects of metal pollution and temperature on metabolism in aquatic ectotherms: implications of global climate change. Climate Research 37 181-201. (doi:10.3354/cr00764)

Spragg J 2018 Feed Grain Supply \& Demand Report 2018. Feed Grain Partnership. (doi:http://www.feedgrainpartnership.com.au/items/1023/FGP\%20Feed\%20Grain\%20Suppl y\%20and\%20Demand\%20Report\%200ct\%202018.pdf)

Stuart KC, Cardilini APA, Cassey P, Richardson MF, Sherwin WB, Rollins LA \& Sherman CDH 2021a Signatures of selection in a recent invasion reveal adaptive divergence in a highly vagile invasive species. Molecular Ecology 30 1419-1434. (doi:10.1111/mec.15601)

Stuart KC, Edwards RJ, Cheng Y, Warren WC, Burt DW, Sherwin WB, Hofmeister NR, Werner SJ, Ball GF, Bateson $M$ et al. 2021b Transcript- and annotation-guided genome assembly of the European starling. BioRxiv 2021.04.07.438753. (doi:10.1101/2021.04.07.438753)

Supek F, Bošnjak M, Škunca N \& Šmuc T 2011 REVIGO Summarizes and Visualizes Long Lists of Gene Ontology Terms. PLOS ONE 6 e21800. (doi:10.1371/journal.pone.0021800)

Tong X-J, López-Soto EJ, Li L, Liu H, Nedelcu D, Lipscombe D, Hu Z \& Kaplan JM 2017 Retrograde Synaptic Inhibition Is Mediated by $\alpha$-Neurexin Binding to the $\alpha 2 \delta$ Subunits of N-Type Calcium Channels. Neuron 95 326-340.e5. (doi:10.1016/j.neuron.2017.06.018)

Turbelin AJ, Malamud BD \& Francis RA 2017 Mapping the global state of invasive alien species: patterns of invasion and policy responses. Global Ecology and Biogeography 26 78-92. (doi:10.1111/geb.12517)

Versluijs M, Turnhout CAM van, Kleijn D \& Jeugd HP van der 2016 Demographic Changes Underpinning the Population Decline of Starlings Sturnus vulgaris in The Netherlands. Ardea 104 153-165. (doi:10.5253/arde.v104i2.a7)

Wada K, Sakaguchi H, Jarvis ED \& Hagiwara M 2004 Differential Expression of Glutamate Receptors in Avian Neural Pathways for Learned Vocalization. The Journal of Comparative Neurology 476 44-64. (doi:10.1002/cne.20201)

Wilson Sayres MA 2018 Genetic Diversity on the Sex Chromosomes. Genome Biology and Evolution 10 1064-1078. (doi:10.1093/gbe/evy039)

Wright B, Farquharson KA, McLennan EA, Belov K, Hogg CJ \& Grueber CE 2019 From reference genomes to population genomics: comparing three reference-aligned reduced- 
bioRxiv preprint doi: https://doi.org/10.1101/2021.08.22.457241; this version posted August 23, 2021. The copyright holder for this preprint (which was not certified by peer review) is the author/funder, who has granted bioRxiv a license to display the preprint in perpetuity. It is made available under aCC-BY-NC-ND 4.0 International license. 